\title{
Habilidades económicas y su relación con la permanencia escolar. Programas de tutoría
}

Rosalía de la Vega Guzmán ${ }^{1}$ y Cinthya Berenice Rodríguez Piedra ${ }^{1}$

\section{Resumen}

Las dificultades de los estudiantes de nivel superior están relacionadas con la falta de habilidades para leer y escribir, pero también con habilidades para la vida como las económicas. El programa de tutoría de la Facultad de Psicología de la Universidad Michoacana de San Nicolás de Hidalgo ha encontrado que el factor económico puede tener implicaciones en este desarrollo escolar. Por lo tanto se desarrolló un estudio para conocer las habilidades económicas de estudiantes de primer año con el fin de tener elementos para proponer el tema de educación económica en el programa de tutorías. Se aplicó una encuesta a 50

1 Universidad Michoacana de San Nicolás de Hidalgo, rosaliadelavega@gmail.com 
estudiantes de dicha facultad que constaba de 10 preguntas organizadas en cinco ejes: consumo, planeación de gasto, ahorro, endeudamiento y permanencia escolar. Los resultados muestran que los participantes presentan pocas habilidades de planeación económica, por lo que sus prácticas de consumo y ahorro pueden tener efectos en algunas de sus actividades escolares.

Palabras clave: habilidades económicas; estudiantes universitarios; tutoría; educación económica.

\section{Abstract}

The difficulties of upper level students are related to the lack of literacy skills, but also with life skills such as economics. The tutoring program of the Faculty of Psychology of the Universidad Michoacana de San Nicolás de Hidalgo has found that the economic factor may have implications in this school development. Therefore, a study was developed to asses the economic abilities of first year students in order to have elements to propose the theme of socioeconomic education in a tutoring program. An interview was applied to 50 students of that faculty that consisted of 10 questions organized around five themes: consumption, spending planning, saving, indebtedness and school permanence. The results show that participants have few economic planning abilities, so 
their economic consumption and saving practices can have an effect on some of their school activities.

Keywords: economic abilities; university students; tutoring; socioeconomic education.

La ANUIES (2000) plantea que el programa de tutoría debe incidir en el abatimiento del rezago, la reprobación y la deserción escolar, poniendo sobre la mesa un reto trascendental para las Instituciones de Educación Superior (IES). Lo anterior puede reflejarse en estudios como el de Albanes, Marques de Sousa y Patta (2015) quienes analizaron 31 publicaciones sobre el tema en el Banco de Tesis de CAPES, SciELO, INDEZPSI y BVS-Psi. En este estudio bilbiométrico se encontró que la mayoría de las investigaciones reportadas indicaron una evaluación positiva de las experiencias de mentoría y tutoría. Asimismo encontraron que muchos de los programas se estructuran comúnmente en dos modalidades: individual y grupal, en las cuales se tratan temas referentes al desarrollo académico de los estudiantes relacionados a los conocimientos disciplinares a los que están dirigidos los programas educativos en las escuelas. Los autores afirman que los programas de tutoría, que se desarrollan tanto en el nivel medio superior como en el superior, están encaminados principalmente en brindar apoyo a los estudiantes para que estos enfrenten sus tareas académicas de forma efectiva, y a la vez puedan 
atender las demandas de la vida universitaria. Estos programas se enfocan principalmente en la atención al desarrollo de competencias escolares útiles en este nivel escolar, como son la escritura y lectura, o las habilidades sociales; por ello, se dice que la acción de educar va más allá de la labor de impartir clases.

Para Hernández y Tobón (2016) los programas de tutoría deben incidir no sólo en los elementos que requiere un estudiante para cumplir con los objetivos académicos que las mismas escuelas les imponen (escritura académica, lectura a profundidad, pensamiento crítico), sino también deben contemplar aspectos más personales como los que contempla el concepto de tutoría socioformativa, entendida ésta como

el proceso por el cual se acompaña, evalúa y apoya a los diferentes actores educativos (estudiantes, docentes, directivos y familiares), para que se logre una formación integral mediante la identificación, argumentación y resolución de problemas del contexto, a través de estrategias como la gestión de conocimiento y la metacognición. (Hernández y Tobón, 2016, p. 35)

En la propuesta de Hernández y Tobón, se hace alusión a la manera en que el concepto de tutoría socioformativa puede servir para atender a aquellos elementos que impactan en la llamada educación integral. Es así que la tutoría socioformativa busca la realización de las personas, el fortalecimiento del tejido social, el desarrollo socioeconómico de las comuni- 
dades en donde se desarrollarán los profesionales, el desarrollo científico, el mejoramiento de la calidad de vida, la sostenibilidad ambiental y el manejo de tecnologías de la información y la comunicación (Maldonado, 2015, en Hernández y Tobón, 2016).

Un concepto diferente que se introduce en esta propuesta de Hernández y Tobón es la del desarrollo de habilidades de emprendimiento, concepto que es utilizado con mayor incidencia en las ciencias administrativas y económicas. Estas habilidades de emprendimiento incluyen la capacidad que tienen o pueden desarrollar los estudiantes para elaborar proyectos empresariales que les permitan aplicar las competencias desarrolladas en su tránsito por las IES.

Sin embargo, en este concepto de emprendimiento, se señala que el alumno requerirá conocer aspectos del mundo económico que regularmente no son del todo claros para los estudiantes por falta de una educación económica que apoye al desarrollo de su comprensión de la realidad económica (Diez-Martínez, 2009). Esta educación puede iniciar con el proceso de socialización económica (Amar, Abello, Denegri, Llanos y Suárez, 2008) pero debe apoyarse en una formación más sistematizada a través de programas educativos destinados para este propósito.

\section{Un programa de tutoría}

El panorama descrito requiere un cambio que posibilite una educación verdaderamente integral. En respuesta a 
ello, las IES en México implementan los programas de tutoría propuestos por la ANUIES. En el caso de la Facultad de Psicología de la Universidad Michoacana de San Nicolás de Hidalgo (UMSNH), se diseñó en 2005 un programa de tutoría que ha tenido como objetivo atender a estudiantes a través actividades tutoriales individualizadas. Posteriormente, en el año 2008, a partir de experiencias y testimonios de profesores tutores y de estudiantes tutorados, se desarrolló y definió la modalidad de tutoría grupal para los estudiantes de primer año, modalidad que se centra en el desarrollo de los grupos, más que en las habilidades escolares.

La decisión se tomó a partir de la consideración de que los mayores índices de reprobación y deserción se presentaban al inicio de la carrera. Así, el objetivo del programa de tutoría grupal de la Facultad de Psicología de la UMSNH se configuró de cara a promover una red de apoyo en los grupos para que puedieran desarrollarse en la vida universitaria de manera óptima, es decir, identificándose como miembros de un grupo, capaces de organizarse, rescatando las cualidades de cada uno en el grupo y respetando sus singularidades, habituados a tomar decisiones y a practicar una comunicación eficaz y asertiva, desarrollando un clima de grupo favorable a los objetivos académicos.

Ante estas necesidades, las temáticas del programa de tutoría grupal se organizaron en 5 ejes específicos: 1) presentación e integración grupal, 2) organización grupal, 3) trabajo en equipo, 4) comunicación asertiva y 5) toma de decisiones. Para su 
evaluación, el programa de tutoría grupal ha venido utilizando reportes cualitativos que elaboran los profesores que fungen como tutores.

Si bien ya se cumplieron 10 años de que el programa dio inicio, actualmente se realiza la actualización del mismo. Parte del trabajo que informa la actualización del programa proviene del análisis de los reportes cualitativos de cinco años atrás, en los que se describen las características y necesidades manifestadas por los grupos. Los resultados de este análisis mostraron diferentes aspectos que pueden afectar al desarrollo escolar, entre ellos la aparición de la vida cibernética (el uso de redes sociales como elementos facilitadores o no de la comunicación), situaciones de acoso y violencia de género $y$, como constante de gran relevancia, la falta de recursos económicos en el estudiante (De la Vega y Cendejas, 2017), temas que ameritarían ser abordador desde la práctica tutorial.

Parecería necesario entonces atender aquellas habilidades que se encuentran al margen de las competencias académicas como el desarrollo de habilidades sociales, base fundamental para el desarrollo de trabajo en equipo, además de aquellas que les serían útiles en su inserción al mundo económico complejo que rodea al desarrollo profesional.

\section{Formación para vida económica}

Partiendo de lo anterior, se puede decir que existen diferentes variables que deben contemplarse en la 
formación universitaria, incluyendo entre ellas los aspectos económicos, que pueden presentarse como una limitante para continuar con la formación educativa, ya sea porque los estudiantes tienen que trabajar para mantener sus estudios o porque las habilidades de organización de los recursos no son desarrolladas en edades tempranas (Diez-Martínez, 2009; Delval, 200; Buckingham, 2013). Así, la economía en general y lo que se refiere a las habilidades económicas en particular, aparece como un factor que influye de forma significativa en el rezago, la reprobación y la deserción escolar (Lugo, 2013).

Actualmente el $20 \%$ del total de la matrícula de los estudiantes de la UMSNH provienen de otros Estados (Hernández, 2018), sin contar a los que llegan del interior de Michoacán. Esta situación hace que los estudiantes de la UMSNH, como otros, se enfrenten al cambio de estar en casa con sus padres sin ser responsables de decisiones económicas trascendentales, al de organizar sus recursos económicos para poder vivir, como, por ejemplo, contemplar el gasto para el transporte público, la bibliografía, la comida, la renta de un espacio para vivir, el ocio, etcétera.

Las características cognitivas de estos estudiantes que ingresan por primera vez a la vida universitaria les permiten presentar un pensamiento hipotéticodeductivo que les brinda la posibilidad de organizar su mundo de manera más compleja a través de formsa de planificar que integran cada vez más variables en conjunto, anticipando los efectos de esta integración 
(Delval, 1994). La mayoría de los estudiantes de primer año universitario se encuentran entre los 17 y los 19 años de edad, lo que permite plantear que tienen experiencias previas respecto a la organización, planeación y toma de decisiones, pero las experiencias económicas pueden ser diferentes, ya que algunos de estos estudiantes, por lo menos en el caso de la UMSNH, viven alejados de sus padres, lo que implica que deben hacerse responsables del manejo de sus recursos, tanto personales como económicos. En muchas ocasiones, los jóvenes se inician en el manejo económico autónomo justo cuando salen de sus casas a estudiar o cuando se casan y deben enfrentar las responsabilidades de mantener una familia (Bedolla, 2014).

La formación universitaria implica una exigencia de autonomía y regulación del estudiante en todos los aspectos, incluyendo el económico. Un estudio de Wang y Xiao (2009) afirma que las habilidades de planeación económica, uso del crédito, habilidades de consumo inteligente, entre otras, son también una pieza clave para la formación universitaria. En esta línea, un estudio desarrollado por Gutiérrez y Delgadillo (2018) muestra que los estudiantes de primer grado de una escuela de nivel superior en Bolivia, presentan significativas limitaciones en su comprensión, aplicación y evaluación de acciones relacionadas al ámbito financiero. Atendiendo a hallazgos como estos, se considera pertinente abordar el ámbito económico en el marco de la intervención tutorial, de modo que se 
pueda facilitar que los estudiantes adquieran conocimientos y habilidades básicas en esta área de la vida.

\section{Método}

Se desarrolló un estudio cuantitativo, transversal, de alcance descriptivo que buscó conocer las habilidades económicas de estudiantes de nivel superior con el fin de tener elementos para proponer el tema de educación socioeconómica en un programa de tutoría.

Para llegar al objetivo planteado se aplicó una encuesta a 50 estudiantes de la Facultad de Psicología de la Universidad Michoacana de San Nicolás de Hidalgo en México. El criterio principal de inclusión de los participantes fue que se encontraran cursando el primer año de formación. Los participantes presentaban una edad promedio de 19.3 años.

El instrumento, que se administró de manera individual, fue un cuestionario que constaba de 10 preguntas cerradas relativas a sus prácticas de consumo, sus habilidades de planeación de gasto, sus prácticas de ahorro, su situación de endeudamiento y la relación entre la planificación económica de sus gastos y su desarrollo escolar. Las preguntas fueron organizadas en cinco ejes: consumo, planeación de gasto, ahorro, endeudamiento y permanencia escolar. La validación del instrumento se desarrolló con un piloteo en el que participaron 25 estudiantes con las mismas características de la población meta. El cuestionario contenía además una ficha sociodemográfica 
para averiguar el estado civil, la actividad laboral y el contexto sociocultural de los participantes. En este apartado del cuestionario, se solicitaba información acerca del nivel escolar de los padres, así como de la ocupación que desarrollaban, tanto la económica como la no económica.

\section{Resultados}

Los participantes presentan características sociales y económicas comunes a los jóvenes que estudian: solo el $4 \%$ de ellos es casado o divorciado, el 94\% es soltero y el $2 \%$ no respondió a la pregunta. En el caso de las actividades económicas que reportan, el 64\% indicó no tener una actividad laboral, el 24\% sí labora y el 12\% no respondió la pregunta.

Respecto al contexto social, académico y económico, los padres de los participantes laboran como empleados (34\%), comerciantes (20\%), practican-tes de un oficio (10\%), profesores (10\%) o profe-sionistas (10\%). En muy pocos casos los padres trabajan como obreros (4\%), agricultores (4\%) 0 campesinos (2\%). El resto de los participantes no respondieron a la pregunta (6\%). En el caso de las madres de los participantes, la mayoría se dedica al hogar (38\%) o al comercio (9\%). 13\% de las madres labora como profesionista, el 15\% como empleada y el $24 \%$ en otras ocupaciones en general.

Como mencionaron Diez-Martínez, Guerra y Sánchez (1998), una parte importante para la construcción 
del conocimiento del mundo económico es el contacto que tengan los niños con las ocupaciones de sus padres. Este conocimiento se obtiene a través de procesos socializadores (Kim, Yang y Lee, 2015; Amar, et al., 2009).

En cuanto a la escolaridad de los padres y de las madres de los participantes, la Tabla 1 presenta su distribución por niveles.

Tabla 1.

Nivel educativo de los padres

\begin{tabular}{lrr}
\hline \multicolumn{1}{c}{ Nivel educativo } & Padre (\%) & Madre (\%) \\
\hline Sin datos & 6 & 2 \\
Básico & 30 & 24 \\
Medio superior & 28 & 32 \\
Técnico & 4 & 0 \\
Superior & 22 & 34 \\
Posgrado & 10 & 8 \\
\hline Total & 100 & 100 \\
\hline
\end{tabular}

Siendo el contexto una fuente de información, es necesario mencionar la forma en la que viven estos jóvenes universitarios. Como menciona Buckingham (2013), las experiencias económicas en las primera etapas de su vida son cruciales para el aprendizaje de estrategias y habilidades para desenvolverse en un mundo económico complejo. Muestra de esto es la 
condición de vida que presentan los jóvenes universitarios que respondieron el cuestionario: el 68\% vive con sus padres, el 14\% con algún familiar, el $12 \%$ vive sólo, el $2 \%$ vive con amigos, el $2 \%$ en casa de estudiantes y el $2 \%$ restante corresponde al participante que no respondió.

Una vez ofrecidos algunos datos sobre el contexto social, cultural y económico de los participantes, pasamos a referir la cantidad de recursos económicos que manejan estos jóvenes a la semana. Para consignar esta información, se les preguntó lo siguiente: Aproximadamente, ¿qué cantidad de dinero recibes a la semana? Las respuestas indican que la mayoría de los estudiantes (36\%) reciben entre $\$ 300$ y $\$ 400$, seguido de los que reciben más de $\$ 500$ (26\%). Solo el $20 \%$ dice disponer en una semana de entre \$100 y \$200; finalmente, el $18 \%$ dice recibir entre $\$ 200$ y $\$ 300$ cada semana.

Aunque un gran número de estudiantes recibe entre $\$ 300$ y $\$ 500$ a la semana, existen jóvenes que deben cubrir los gastos que genera la vida universitaria con $\$ 100$ a la semana. Sin embargo, es necesario analizar los tipos de gasto que reportan estos estudiantes, considerando aquellos gastos a los que les dan más prioridad y aquellos que resultan inevitables. Para indagar lo anterior se formularon las siguientes preguntas: ¿Cuáles son los gastos que los jóvenes universitarios identifican?, y ¿a cuáles les dan mayor prioridad estos estudiantes? Las respuestas obtenidas a través del cuestionario se presentan en función de los 
ejes preestablecidos en el diseño del instrumento utilizado.

\section{Consumo}

Para abordar este eje, la encuesta indagó acerca de la distribución de sus recursos económicos en forma de prioridades. Para ello, se les pidió que de una lista previa jerarquizaran a lo que le dan más prioridad en el momento de organizar sus recursos económicos. En la lista se incluyeron rubros como: educación, salud, alimentación, ropa y calzado, renta de vivienda, servicios de vivienda, deudas transporte, ahorro, ocio y diversión. A continuación se presentan los resultados de esta jerarquización de administración de gasto en porcentaje de frecuencia de respuestas.

En la Tabla 2 se puede observar que los participantes indican priorizar el gasto en alimentación a la hora de organizar sus recursos económicos (16.8\%), seguido del transporte (11.7\%). Por ello, se puede inferir que probablemente el monto de recursos económicos que recibe la mayoría de estos estudiantes es apenas suficiente para este tipo de gasto. El rubro de educación fue jerarquizado en igual proporción en el $2^{\circ}$ nivel $(6.6 \%)$ y en el $4^{\circ}$ nivel (6.6\%), lo que podría estar también relacionado con la cantidad monetaria que estos jóvenes tienen para solventar sus estudios. Desde la percepción de los participantes los rubros de servicios de vivienda (10.2\%) o renta de vivienda (13.9\%) son los menos prioritarios a la hora de orga- 
nizar su gasto, lo cual es congruente con el el hecho de que la mayoría de estos jóvenes aún viven con sus padres y no se hacen cargo de este tipo de gasto. Esto concuerda con el estudio de Corominas (2001) que indica que la mayoría de los jóvenes universitarios que participaron en su estudio son financiados por sus padres, y por lo tanto una parte importante de su gasto es dirigido a actividades de ocio.

Tabla 2.

Jerarquía de administración del gasto

\begin{tabular}{rlr}
\hline Posición & \multicolumn{1}{c}{ Tipo de gasto } & $\%$ \\
\hline $1^{\circ}$ & Alimentación & 16.8 \\
$2^{\circ}$ & Educación & 6.6 \\
$3^{\circ}$ & Transporte & 11.7 \\
$4^{\circ}$ & Educación & 6.6 \\
$5^{\circ}$ & Salud & 8.0 \\
$6^{\circ}$ & Ropa y calzado & 6.6 \\
$7^{\circ}$ & ocio y diversión & 5.8 \\
$8^{\circ}$ & Ahorro & 6.6 \\
$9^{\circ}$ & Deudas & 7.3 \\
$10^{\circ}$ & Servicios de vivienda & 10.2 \\
$11^{\circ}$ & Renta de vivienda & 13.9 \\
\hline
\end{tabular}

Finalmente, el menor valor jerárquico fue asignado al rubro de ocio y diversión, presentándose sus 
respuestas en la posición 7 con el 5.8\%. Para los estudiantes universitarios las actividades de ocio son importantes probablemente como menciona Rodríguez (1999) por proveerles de un estilo de vida específico y transitorio del resto de la población en general, en donde este tipo de actividades fomenta el desarrollo de ciertos valores y actitudes correspondiente a la etapa de desarrollo en la que se encuentran.

Planeación de gasto

Los resultados muestran que los participantes presentan pocas habilidades de planeación económica, por lo que sus prácticas de consumo pueden intervenir en algunas de sus actividades escolares. En el cuestionario se les preguntaba si hacían registro de sus gastos en rubros específicos (deudas, gastos en general, ingresos, ahorro). El análisis de frecuencias de respuestas muestran que en el caso del tema de las deudas el $7.14 \%$ reporta registrarlas; en el caso de los gastos en general el $28.57 \%$ de los participantes afirman llevar un registro. Los ingresos los registra solo el 17.4\%. Las prácticas de ahorro son registradas por el $22.6 \%$ de los participantes y el $24.29 \%$ menciona no llevar ningún registro de estos rubros. Esto se relaciona con lo reportado por Estrada, Moya, Adanda y Rivera (2017), cuando mencionan que los jóvenes tienen escaso conocimiento sobre el manejo de sus finanzas personales, lo que tiene implicaciones en sus habilidades financieras. 
En cuanto a las formas en que los estudiantes planean sus gastos; el $11.9 \%$ de los participantes reporta hacerlo libremente, el $9.5 \%$ por escrito y el $78.6 \%$ dice hacerlo de forma mental, lo cual coincide con los resultados que muestra la Procuraduría Federal del consumidor (PROFECO) en la encuesta que realizó en 2010 sobre Presupuesto y riesgos financieros, en donde se señala que el $62 \%$ de los 1,325 encuestados llevan un registro mental de sus egresos, lo que ha tenido repercusiones en su forma de gastar (46\%). Lo anterior pone de relieve la importancia de la educación económica en cualquier nivel educativo, pero con mayor énfasis en el nivel superior, ya que están prontos a integrarse al mundo laboral y con ello a enfrentarse a diferentes instituciones financieras. Esta información es consistente con lo descrito en la síntesis de resultados de la Encuesta de Cultura financiera de los Jóvenes en México (2014), en la cual se reporta que solo el $22 \%$ de los encuestados de entre 15 y 29 años de edad lleva algún tipo de presupuesto (Banamex-UNAM, 2014).

\section{Ahorro}

En cuanto a las prácticas de ahorro de los jóvenes universitarios, se preguntó a los participantes el porcentaje de su presupuesto que destinan al ahorro. Las respuestas señalan que el 32\% ahorra cantidades mínimas: alrededor del $5 \%$ de su presupuesto, pero la mayoría a de ellos dice ahorrar cada semana (56\%) y el lugar que usan para guardar su dinero es su casa 
(76\%), en contraste con los que usan el banco como una forma de ahorro (16\%). Lo anterior puede compararse con los resultados presentados en el reporte Nacional de Inclusión Financiera (2016) donde se señala que el $58 \%$ de los encuestados dicen ahorrar, pero sólo el $14 \%$ lo hace en una institución financiera. Además, los efectos de las escasas habilidades de ahorro también pueden ser un reflejo del entrenamiento o experiencia que tengan estos jóvenes al elaborar un presupuesto de ingresos y gastos cotidianos, ya que para realizar esta labor las estrategias de ahorro son necesarias (Estrada et al., 2017).

\section{Endeudamiento}

Respecto a la categoría de endeudamiento los resultados de la encuesta muestran que los universitarios participantes practican el endeudamiento: el 30\% reporta que ha tenido que pedir dinero prestado específicamente para no dejar sus estudios. Los participantes refieren que han tenido que solicitar préstamos para ir un día o más a la escuela, y aunque el porcentaje de estudiantes que refiere este tipo de préstamo refleja la situación de menos de la mitad de los encuestados (36\%), esto supone una señal de alerta en cuanto a la continuidad de sus estudios. Estos datos se relacionan con lo reportado por Rodríguez (2000) en un estudio realizado en Costa Rica, cuando indica que el factor que más incide en el abandono de los estudios es el socioeconómico. 
Aunque el tipo de préstamos que realizan los participantes no es del tipo institucional en el sentido de que no lo contratan con instituciones financieras, parece conveniente considerar las formas en que los jóvenes adquieren experiencia en cuanto a créditos, ya que, en la actualidad, muchas instituciones financieras se están acercando a las universidades para ofrecer, con pocos requisitos, líneas de crédito a los estudiantes (Domínguez, 2017). Habría que considerar además que, habitualmente, los jóvenes no cuentan con información suficiente sobre las reglas de los servicios que ofrecen estas instituciones financieras (Estrada et al., 2017).

\section{Conclusiones}

La formación de los estudiantes dentro de las IES debe fortalecerse de tal manera que pueda dar una garantía más o menos certera de una educación integral. Los programas de tutoría han estipulado la importancia de la misma y se han desarrollado estrategias como la tutoría socioformativa, que propone vías más concretas. Sin embargo, la realidad económica a la que se enfrentarán los egresados de las IES es tan compleja y demandante que difícilmente se encuentran preparados para ella, por lo que es vital ir sumando a la propuesta de formación integral una educación económica que apoye el desarrollo de su pensamiento económico y que puede repercutir en la creación o dominio de estrategias económicas, además de las habilidades y 
actitudes necesarias para transitar en la economía desde una perspectiva autónoma.

Los resultados presentados de este estudio abren un camino para seguir indagando, de manera más específica y con una población más amplia, las características de las habilidades y conocimientos económicos que les serán útiles a los universitarios tanto en el área profesional como en la personal.

Es necesario mencionar que existieron algunas limitaciones en este estudio de tipo metodológico, entre ellas la estructura del instrumento, que no permitió conocer detalles más específicos de algunas repuestas, y la cantidad de estudiantes que participaron.

A pesar de lo anterior, las aportaciones empíricas de este estudio servirán para elaborar una propuesta a incluir en el programa de tutorías de la Facultad de Psicología de la UMSNH, en la forma de una línea de acción sobre educación económica. Contar con un programa de tutoría que contemple dicho contenido e impacte en la mejora de la vida económica estudiantil será un elemento que incida en el cumplimiento del objetivo de la formación integral.

\section{Referencias}

Amar, J., Abello R., Denegri, M., Llanos, M. y Suárez, R. (2008). Estrategias de socialización económica en padres de familia. Economía del Caribe, 1, 156-183. 
ANUIES (2000). Programas Institucionales de Tutoría.

Una propuesta de la ANUIES para su organización y funcionamiento en las instituciones de educación superior. México: ANUIES.

Albanaes, P., Marques, F. y Patta, M. (2015).

Programas de tutoría en universidades de Brasil:

un estudio bibliométrico. Revista de Psicología.

33(1), 21-56.

Banamex-UNAM (2014). Cultura Financiera de los

Jóvenes en México. Síntesis de resultados.

México: Banamex. Recuperado de

https://www.banamex.com/es/conoce_banamex/q

uienes_somos/prensa/pdf/

book_brujula_digital_2014.pdf

Bedolla, Y. (2014). Prácticas de consumo en

adolescentes de un contexto semirural y su implicación con sus elecciones vocacionales

[Tesis de licenciatura]. Morelia: Universidad Michoacana de San Nicolás de Hidalgo.

Buckingham, D. (2013). La infancia materialista. Crecer

en la cultura consumista. Madrid: Morata.

Consejo Nacional de Inclusión Financiera (2016).

Reporte Nacional de Inclusión Financiera 7.

Recuperado de http://www.cnbv.gob.mx/Inclusi

\%C3\%B3n/Documents/Reportes\%20de\%20IF/

Reporte\%20de\%20Inclusion\%20Financiera

\%207.pdf

Corominas, E. (2001). La transición a los estudios

universitarios. Abandono o cambio en el primer 
año de universidad. Revista de investigación

Educativa, 19(1), 125-151.

De la Vega, R. y Cendejas, O. J. (2017). Las TiCs como apoyo para el diagnóstico específico en la acción tutorial entre pares. Ponencia presentada en el $5^{\circ}$ Encuentro Regional de Tutoría. Red de Tutoría de la Región Centro Occidente de la ANUIES.

Morelia (México), 30 de octubre-1 de noviembre.

Delval, J. (1994). El desarrollo humano. Madrid: Siglo $\mathrm{XXI}$.

Delval, J. (2007). Aspectos de la construcción del conocimiento sobre la sociedad. Educar Curitiba, 30, 45-64. Recuperado de https://www.scielo.br/pdf/er/n30/a04n30

Diez Martínez, E., Guerra, E. y Sánchez, M. (1998). Concepciones de los niños mexicanos sobre el trabajo de sus padres y el de otras personas: Los mecanismos de obtención del empleo y las fuentes de la remuneración del mismo. Revista Latinoamericana de Pensamiento y Lenguaje. 6(2), 39-52.

Diez-Martínez, E. (2009). La alfabetización socioeconómica y financiera y la educación para el consumo sostenible en México: algunas reflexiones desde la psicología y la educación. CPU-e. Revista de Investigación Educativa, 8. Recuperado de http://www.uv.mx/cpue/num8/opinion/alfabetizacio $\underline{\text { n.html }}$ 
Domínguez, F. (2017, 31 de julio). ¿De verdad necesitas una tarjeta de crédito para estudiantes?

FORBES México. Recuperado de

https://www.forbes.com.mx/de-verdad-necesitas-

una-tarjeta-de-credito-para-estudiantes/

Estrada, A., Arévalos, P., Moya, S., Aranda, C. y Rivera,

S. (2017). Propuesta de cuestionario para medir el

nivel de conocimiento sobre las finanzas

personales de jóvenes universitarios. Global

Business Administration Journal, 1(2), 56-64.

Gutiérrez, O. y Delgadillo, J. (2018). La educación financiera en jóvenes universitarios del primer ciclo de pregrado de la Universidad Católica Boliviana" San Pablo", Unidad Académica Regional de Cochabamba. Revista Perspectivas, 41, 33-72.

Hernández, A. (2018, 19 de marzo). En promedio, 20\% de matrícula de la UMNSH corresponde a foráneos. Mi Morelia.com. Recuperado de https:// www.mimorelia.com/promedio-20-matricula-laumsnh-corresponde-foraneos/

Hernández, V. y Tobón, S. (2018). La tutoría socioformativa en la educación superior. Revista Docencia e Investigación, 1(27), 33-58.

Kim, C., Yang, Z. y Lee, H. (2015). Parental style, parental practices, and socialization outcomes: An investigation of their linkages in the consumer socialization context. Journal of Economic 
Psychology, 49, 15-33.

doi:10.1016/j.joep.2015.03.006

Lugo, B. (2013). La deserción estudiantil: ¿Realmente es un problema social? Revista de postgrado FACE-UC, 7(12), 289-309.

Otto, A. (2013). Saving in childhood and adolescence: Insights from developmental psychology.

Economics of Education Review, 33, 8-18.

PROFECO (2010). Difunde PROFECO resultados de su encuesta sobre presupuesto y riesgos financieros. Recuperado de https://www.profeco.gob.mx/prensa/prensa10/octu bre10/bol136.asp

Rodríguez, J. y Agulló Tomás, E. (1999). Estilos de vida, cultura, ocio y tiempo libre de los estudiantes universitarios. Psicothema, 11(2), 247-259.

Rojas Rodríguez, M. (2000). La deserción escolar en costa rica: Un estudio de causas y consecuencias en una institución educativa. Diálogos Revista Electrónica de Historia, 1(4).

Wang, J. y Xiao, J. J. (2009). Buying behavior, social support and credit card indebtedness of college students. International Journal of Consumer Studies, 33(1), 2-10. 


\section{(c) (1) (9)}

Esta obra está bajo una

\section{Licencia Creative Commons Atribución-NoComercial-} Compartirlgual 4.0 Internacional

Usted es libre de compartir o adaptar el material en cualquier medio o formato bajo

las condiciones siguientes: (a) debe reconocer adecuadamente la autoría, proporcionar un enlace a la licencia e indicar si se han realizado cambios; (b) no puede utilizar el material para una finalidad comercial y (c) si remezcla, transforma

o crea a partir del material, deberá difundir sus contribuciones bajo la misma licencia que el original.

Resumen de la licencia

https://creativecommons.org/licenses/by-nc-sa/4.0/deed.es_ES

Texto completo de la licencia https://creativecommons.org/licenses/by-nc-sa/4.0/legalcode 